\title{
Use of a transposon (Tndif) to obtain suppressing and nonsuppressing insertions of the dif resolvase site of Escherichia coli
}

\author{
Peter Kuempel, ${ }^{1}$ Andrias Hogaard, Mette Nielsen, Olagappan Nagappan, and Marianne \\ Tecklenburg \\ Department of Molecular, Cellular, and Developmental Biology, University of Colorado, Boulder, Colarado 80309 USA
}

\begin{abstract}
The dif locus is a RecA-independent recombination site, located in the terminus region of the chromosome of Escherichia coli. This site functions to reduce circular dimer chromosomes to monomers before cell division. Strains lacking this site exhibit the Dif phenotype, in which a fraction of the cells form extended filaments with abnormal nucleoids, and the SOS system is induced. We have used a transposon (Tndiff, as well as linear transformation, to position dif in 19 locations around the chromosome. All of the suppressing insertions that we obtained were within $10 \mathrm{~kb}$ of the normal site, even in strains in which the normal symmetry between the origin of replication and dif had been altered by $200 \mathrm{~kb}$. We also observed that the nonsuppressing insertions in the terminus region became suppressing if a deletion occurred that extended from the ectopic site up to or past the normal location of dif. We propose that dif is normally located at the center of converging polarities in the terminus region and that deletions that restore suppression do so by placing ectopic sites once again at the center of this polarity. Similar results and conclusions are described in this issue.
\end{abstract}

[Key Words: E. coli; chromosome; terminus; resolvase; dif]

Received January 11, 1996; revised version accepted March 21, 1996.

The dif locus of Escherichia coli is a RecA-independent recombination site that is related in sequence and function to the cer resolvase site of pColE1 (Blakely et al. 1991; Clerget 1991; Kuempel et al. 1991). dif and cer contain a similar core sequence, which is the binding site for the XerC and XerD resolvase proteins (Blakely et al. 1993). Similar to cer, dif appears to function as a resolvase site, which reduces circular, dimeric bacterial chromosomes to monomers before cell division. These dimers presumably arise from recombination between growing daughter chromosomes / sister chromatid exchange) in a fraction of the cells (Forro and Wertheimer 1960; Van Tubergen and Setlow 1960).

The dif site is located in the middle of the terminus region, directly opposite the origin of replication (ori $C$; Fig. 1). Based on the physical map of the wild-type $E$. coli chromosome, the clockwise and counterclockwise distances from oriC to dif are 2323 and $2350 \mathrm{~kb}$, respectively (Kohara 1987; Rudd 1992 and pers. comm.). Consequently, dif is one of the last loci to be replicated during the replication cycle. This is appropriate for its function, because newly replicated dif sites would provide a convenient location for recombination events that

${ }^{1}$ Corresponding author. resolve dimers. These dimers must be resolved correctly or subsequent nucleoid segregation and cell division will be affected. The Dif phenotype is consistent with this model. In cultures of dif mutants, a fraction of the cells form extended filaments $(\leqslant 50 \mu \mathrm{m}$ long) with abnormal nucleoids, and the SOS DNA repair system is induced (Blakely et al. 1991; Kuempel et al. 1991). These effects are eliminated by suppressing insertions (Tecklenburg et al. 1995). It should also be noted that the largest DNA molecules detectable by viscoelastometry in stationaryphase dif cells were almost twice the size observed in $\mathrm{dif}^{+}$cells (B. Alicke and E. Uhlenhopp, pers. comm.).

We recently demonstrated that proper dif function could be provided by a 33-bp sequence (Tecklenburg et al. 1995) that contained the binding sites for XerC and XerD (Blakely et al. 1993). Similar results have been reported by Leslie and Sherratt (1995). Flanking DNA was not required, and the 33 -bp sequence was sufficient to suppress the Dif phenotype of deletions that removed either 12 or $173 \mathrm{~kb}$ of terminus region DNA (Tecklenburg et al. 1995). The location of the site was important for suppression, because insertions elsewhere in the chromosome (Leslie and Sherratt 1995), or in the terminus region but $118 \mathrm{~kb}$ from the normal site (Tecklenburg et al. 1995), failed to suppress the Dif phenotype. However, these insertions still exhibited a high frequency of 


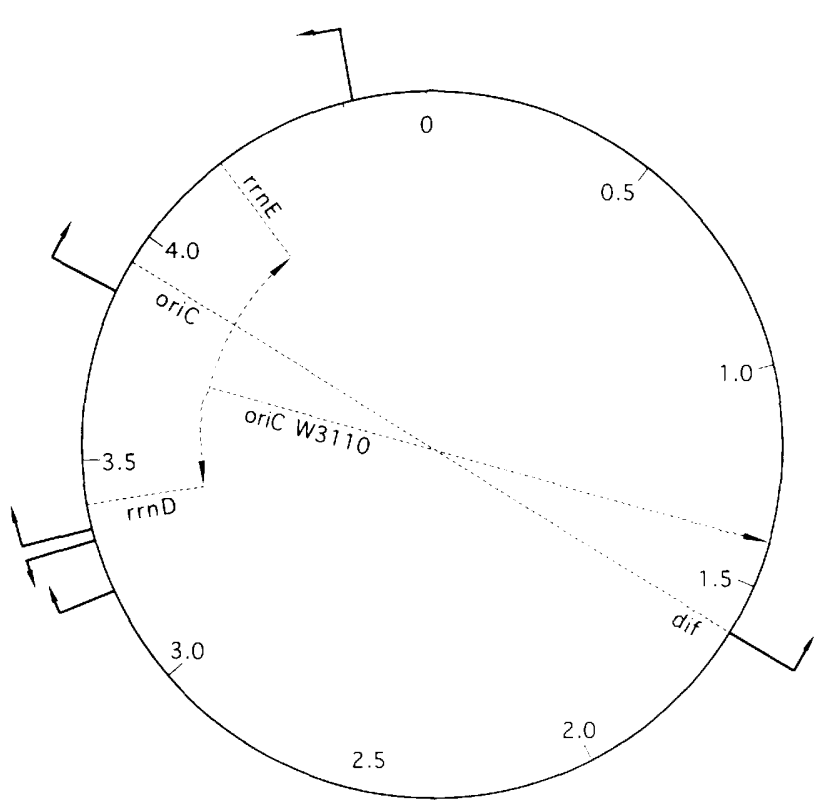

Figure 1. The E. coli chromosome, based on the physical coordinates of EcoMap6 (K. Rudd, pers. comm.; also see EcoMap5, Rudd 1992). The physical map is indicated in megabases |Mb| and starts at 0 min of the genetic map. The arrows outside the circle indicated the location and orientation of five nonsuppressing Tndif insertions. The origin of replication $(o r i C)$ is at $3952 \mathrm{~kb}(84.6 \mathrm{~min})$ in a wild-type chromosome, and dif is directly opposite at $1599 \mathrm{~kb}$ (34.2 min). In strains with IN (rmD-rmE) 1, such as W3110, the origin is shifted and the region opposite oriC is at $1400 \mathrm{~kb}(30.0 \mathrm{~min})$. The Tndif insertions are $\Omega(3205 \mathrm{~kb}:$ Tndif $\rightarrow \mid 3478, \Omega(3321 \mathrm{~kb}::$ Tn $\leftarrow$ diff $3477, \Omega(3335 \mathrm{~kb}::$ Tndif $\rightarrow \mid 3486$, $\Omega(3856 \mathrm{~kb}: \mathrm{Tn} d \mathrm{fif} \rightarrow \mid 3485$, and $\Omega(4519 \mathrm{~kb}: \mathrm{Tn} \leftarrow$ dif $) 3479$.

RecA-independent recombination with a dif-containing plasmid. Suppression was also obtained by an insertion, at the dif location, of the loxP site of bacteriophage P1 (Leslie and Sherratt 1995) and of psi, which is a cer-like site in pSC101 (Cornet et al. 1994). The cer site did not suppress the Dif phenotype (Leslie and Sherratt 1995).

The properties and interactions of dif sites provide a novel means to study chromosome structure and events during the completion of the replication cycle, because suppression of the Dif phenotype has only been obtained by insertion of resolvase sites at the normal dif location. Conversely, we also observed that an ectopic site located $118 \mathrm{~kb}$ from the normal dif site became fully functional if the DNA between it and the normal dif location was deleted (Tecklenburg et al. 1995). It was uncertain how the correct function of the dif site was influenced by its position with respect to oriC, as well as its requirements for flanking DNA sequences.

To isolate a variety of insertions, we have constructed a dif transposon. This has been used to obtain insertions at many locations, both in the terminus region and elsewhere around the chromosome. All of the suppressing insertions were clustered near the normal location of dif, although they were isolated in derivatives of strain W3110. These strains were chosen because they contain inversion IN(rrnD-rrnE)1 (Hill and Harnish 1981; Fig. 1) and dif is no longer directly opposite oriC. The insertions have also been used to construct additional deletions and inversions in the terminus region. We report here the results of these studies and discuss a model for our results.

Cornet et al. (this issue) describe different procedures used to study the requirements for a functioning dif site, and their results and conclusions are similar to those reported here.

\section{Results}

The purpose of these experiments was to identify regions in which dif insertions occur that are able to suppress the Dif phenotype. Most of the ectopic sites that we report here were obtained by means of a dif transposon (Tndif). This is different from dif insertions characterized previously by us and others (Cornet et al. 1994; Leslie and Sherratt 1995; Tecklenburg et al. 1995; see also Cornet et al., this issue). In those studies, dif was inserted into targeted sites by construction of appropriate plasmids, and homologous recombination was used to achieve integration. These strains were then tested to determine whether suppression occurred. By using a dif transposon we adopted a different approach. We planned to construct a library of random insertions. Next, an indicator would be used to identify sites that provided suppression, which could then be mapped to identify their locations.

\section{Suppressing insertions of the dif transposon}

The dif transposon that we constructed (Tndif; Fig. 2) contained a 231-bp sequence from the dif locus (Kuempel et al. 1991). Although we observed previously that a 33-bp sequence suppressed the Dif phenotype as well as the 231-bp sequence (Tecklenburg et al. 1995), we recently noted that the longer sequence gave a higher frequency of recombination between chromosomal sites, at least in some situations (P. Kuempel, unpubl.). Because we intended to use dif insertions to cause chromosome rearrangements and deletions, we have concentrated on insertions of the 231-bp sequence. Tndif was derived from pNK2859 (Kleckner et al. 1991), which encodes a Tn 10 transposase with altered target specificity and consequently inserts into a wider range of sites. Expression of the transposase gene is controlled by the Ptac promotor.

Most of our library constructions and tests for suppression have been done in strains derived from W3110. We chose these strains because they contain the IN/rmDrrnE)1 inversion (Hill and Harnish 1981). In strains with the wild-type chromosome structure, the location of dif on the physical map is at $1599 \mathrm{~kb}$ (K. Rudd, pers. comm.), which is directly opposite oriC (Fig. 1). In strains with IN $(r m D-r r n E \mid 1$, the position directly opposite oriC is shifted $200 \mathrm{~kb}$, and it occurs at $1400 \mathrm{~kb}$. Our rationale for using these strains was that it would simplify determin- 
Figure 2. Plasmid pHN13 and Tndif. The 231-bp dif sequence and kan are located between the $70-\mathrm{bp}$ inserted repeats (IR). The gene for the transposase with altered target sepcificity is controlled by Ptac and is located outside the Tndif transposon. Only the relevant restriction enzyme sites are shown: BamHI $|O|$; HindIII $\langle\triangle|$; PstI $|O|$; $K p n I(\square)$. The transpson contains no sites for EcoRI, EcoRV, BglI, or PvuII.

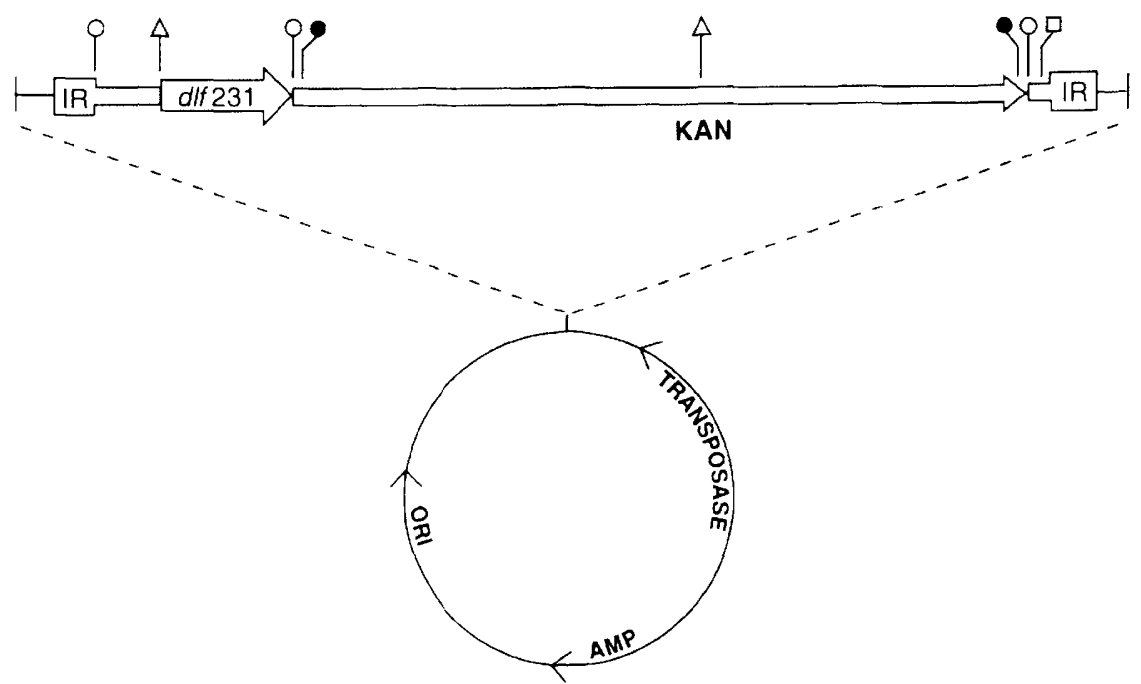

ing whether suppression by ectopic dif sites was influenced by position opposite the origin, or whether it depended on the context of the site.

After induction of transposition in PK3471, bacteriophage P1 was used to transduce the library of Tndif insertions into an indicator strain. Transduction ensured that single insertions were being studied and was usually sufficient to eliminate pHN13, although $50 \%$ of the $\mathrm{Km}^{\mathrm{r}}$ transductants remained $\mathrm{Ap}^{\mathrm{r}}$. The indicator strain PK3032 was derived from W3110, and it contained a 1.3$\mathrm{kb}$ dif deletion ( $\Delta$ dif::cam2771) and a sfiA::lac reporter gene (Lin and Little 1988). Because sfiA is induced in dif mutants by SOS induction (Kuempel et al. 1991; Tecklenburg et al. 1995), unsuppressed colonies were red on MacConkey-lactose agar. Suppressed colonies were readily identified by their white color.

At least $15 \%$ of the $\mathrm{Km}^{\mathrm{r}}$ transductants from various libraries were white on MacConkey-lactose agar, and the cells in those colonies did not exhibit the filamentation typical of dif mutants. We examined a number of pink colonies in an attempt to recover clones with intermediate levels of suppression and SOS induction, but restreaking invariably gave red colonies with filamen- tous cells or white colonies with normal cells. Suppressing insertions were initially mapped by transduction to determine their location. Forty suppressed strains obtained from a series of libraries were tested, and in all of them the Tndif insertion was tightly linked $1>90 \%$ cotransduction) to a spectinomycin resistance gene present at dif.

Seven of the suppressing insertions were mapped (Fig. 3 ) by Southern hybridization in which dif DNA was used as the probe. The particular restriction fragments in which an insertion had occurred could be readily identified on the physical map (Rudd 1992, and pers. comm.), because of an increased size of $1.9 \mathrm{~kb}$ in an overlapping set of EcoRI, EcoRV, BglI, and PvuII fragments. The location and orientation could be determined from the Pst I and KpnI digestions, which cut in the dif transposon (Fig. 2). An example of the full designation used for these insertions is $\Omega(1589 \mathrm{~kb}:: \leftarrow$ Tndif $\mid 3476$. This indicates that the insertion $(\Omega)$ of the Tndif transposon occurred at $1589 \mathrm{~kb}$, that the dif sequence has the same orientation as wild type $\mid \leftarrow l$, and that the insertion was isolated in strain PK3476. Insertions are identified in this fashion in the figure legends and in Table 1 . In the text and figures,

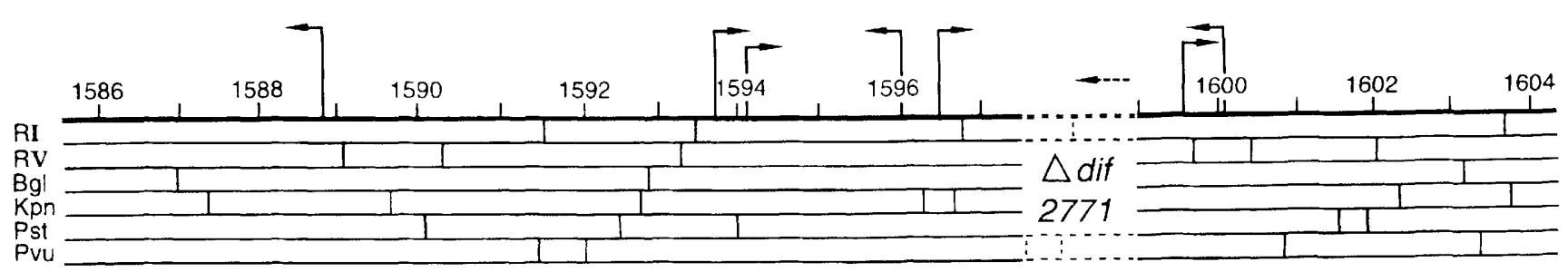

Figure 3. Physical map of the region surrounding dif. The arrows indicate the locations and orientation of seven suppressing insertions mapped by Southern hybridizations. These were isolated in PK3032, in which $\Delta$ dif::cam2771 extends from 1597.7 to 1599 $\mathrm{kb}$. The broken arrow indicates the orientation of wild-type dif. The indicated restriction enzyme sites are from EcoMap6 (K. Rudd, pers. comm.) These sites are, from top to bottom, EcoRI, EcoRV, Bgll, KpnI, PstI, and PvuII. The broken lines in $\Delta$ cam::2771 indicate the EcoRI and PvuII sites in the chloramphenicol resistance gene. The full designations of the Tndif insertions, from left to right, are $\Omega(1589 \mathrm{~kb}::$ Tn $\leftarrow$ dif $\mid 3476, \Omega(1594 \mathrm{~kb}::$ Tndif $\rightarrow \mid 3497, \Omega(1594 \mathrm{~kb}::$ Tndif $\rightarrow \mid 3516, \Omega(1596 \mathrm{~kb}::$ Tn $\leftarrow$ dif $\mid 3518, \Omega(1596 \mathrm{~kb}::$ Tndif $\rightarrow \mid 3504$, $\Omega(1600 \mathrm{~kb}::$ Tndif $\rightarrow \mid 3517$, and $\Omega(1600 \mathrm{~kb}::$ Tn $\leftarrow$ dif $) 3503$. 
Table 1. Genotypes of relevant bacterial strains

\begin{tabular}{|c|c|}
\hline Strain & Genotype \\
\hline PK2649 & $\operatorname{trpR} \operatorname{trp} A 9605$ his-29 ilv arg-427 thy $A$ deoB or deoC tsx IN(rrnD-rrnE)1 $\Delta(\arg F-\operatorname{lac}) 205 \lambda s f i A::$ lacZ Ind $^{-}$ \\
\hline PK2771 & $\begin{array}{l}\text { recB21 recC22 sbcB15 sbcC thi-1 thr-1 leuB6 lacY1 mtl-1 xyl-5 ara-14 galK2 hisG4 proA2 rpsL31 txs-33 supE44 } \\
\quad \Delta d i f: \text { cam2771 }\end{array}$ \\
\hline PK3032 & as PK2649, but $\Delta$ dif::cam2771 \\
\hline PK3269 & as PK2649, but xerC::camY17 recD::Tn10 $\Delta$ dif ::spc3269 \\
\hline PK3270 & $\begin{array}{l}\text { recB21 recC22 sbcB15 sbcC thi-1 thr-1 leuB6 lacY1 mtl-1 xyl-5 ara-14 galK2 hisG4 proA2 rpsL31 tsx-33 supE44 } \\
\quad \text { xerC::camY17 } \Delta \text { dif::spc3269 }\end{array}$ \\
\hline PK3321 & as $\mathrm{PK} 3270$, but $\Omega(1399 \mathrm{~kb}:: \leftarrow$ dif-kan $) 3321$ \\
\hline PK3381 & as $\mathrm{PK} 3270$, but $\Omega(1399 \mathrm{~kb}::$ dif $\rightarrow$ kan $\mid 3381$ \\
\hline PK3436 & as $\mathrm{PK} 2649$, but $i v^{+} \mathrm{xerC}^{+} \Delta \mid \Omega(1399 \mathrm{~kb}:: \leftarrow$ dif-kan $) 3321-$ dif $^{+} \mid 3436$ \\
\hline PK3437 & as PK2649, but $\mathrm{il}^{+}{ }^{+} \mathrm{xerC} C^{+} \mathrm{IN}\left[\Omega\left(1399 \mathrm{~kb}::\right.\right.$ dif $\rightarrow$ kan $\mid 3381-$ dif $^{+} \mid 3437$ \\
\hline PK3468 & IN $(r r n D-r r n E) 1$ lacI ${ }^{\mathrm{Q}}$ L8 $\Delta$ dif::cam2771 \\
\hline PK3471 & PK3468/pHN13 \\
\hline PK3476 & as $\mathrm{PK} 3032$, but $\Omega(1589 \mathrm{~kb}: \mathrm{Tn} \leftarrow \operatorname{dif}) 3476$ \\
\hline PK3483 & $\mathrm{IN}(r r n D-r m E) 1$ recD $::$ mini-tet $x e r C:: t m p$ \\
\hline PK3512 & PK3468 xerC::tmp/pHN13 \\
\hline PK3693 & as PK2649, but $i l_{V^{+}} \Delta[\Omega(1399 \mathrm{~kb}:: d i f \rightarrow$ kan $\mid 3381-\Omega(1632 \mathrm{~kb}::$ Tndif $\rightarrow \mid 3646) 3693$ \\
\hline PK3714 & as PK2649, but $i l^{+} \Delta[\Omega(1596 \mathrm{~kb}::$ Tndif $\rightarrow \mid 3504-\Omega(1632 \mathrm{~kb}::$ Tndif $\rightarrow \mid 3646 / 3714$ \\
\hline PK3742 & as PK2649, but $i V^{+}$xerC ${ }^{+} \Delta\left[\Omega(1487 \mathrm{~kb}::\right.$ Tn $\leftarrow$ dif $) 3645-$ dif $^{+} \mid 3724$ \\
\hline PK3751 & as PK2649, but ilv ${ }^{+}$xerC $C^{+} \Delta[\Omega(1487 \mathrm{~kb}::$ Tn $\leftarrow$ dif $\mid 3645-\Omega(1600 \mathrm{~kb}::$ Tn $\leftarrow$ dif $\mid 3503] 3751$ \\
\hline
\end{tabular}

Not all Tndif kan insertions are included here. The five Tndif insertions outside the terminus region, which were isolated in PK3032, are described in Fig. 1. The seven suppressing insertions, which were isolated in PK3032, are described in Fig. 3. Five nonsuppressing insertions in the terminus region are shown in Fig. 4 and are described in more detail in Results. $\Omega(1589 \mathrm{~kb}::$ Tn $\leftarrow$ dif) 3476 designates an insertion $(\Omega)$ of Tndif at $1589 \mathrm{~kb}$ in EcoMap6 (Rudd 1993), in which $\leftarrow$ dif has the same orientation as $\leftarrow$ dif ${ }^{+}$, and the insertion was first isolated in PK3476.

$\Delta[\Omega(1487 \mathrm{~kb}:: \mathrm{Tn} \leftarrow$ dif $) 3645-\Omega(1600 \mathrm{~kb}::$ Tn $\leftarrow$ dif $|3503| 3751$ designates $د 3751$, formed by XerC-dependent recombination between dif insertions at 1487 and $1600 \mathrm{~kb}$, which were arranged as direct repeats. Strain construction for isolation of deletions from double dif strains is described in Materials and methods.

insertions are simply identified by the kilobase coordinates of the insertion site.

We have demonstrated that the high frequency of suppressing insertions $(\sim 15 \%)$ in our libraries was attributable to the increased growth rate provided by these insertions. For example, we have constructed libraries in a xerC::tmp mutant (PK3512), in which insertions near dif should provide no growth advantage. On transduction to the indicator strain PK3032, only $1 \%$ (2 of 180 ) of the transductants exhibited the suppressed phenotype. These suppressing insertions were tightly linked to dif. We have also conducted reconstruction experiments using mixed cultures of $\mathrm{xerC}^{+}$strains containing suppressing and nonsuppressing insertions. Suppressing insertions were enriched $\sim 20$-fold after bacterial growth and transduction.

\section{Nonsuppressing insertions of the dif-transposon}

Although libraries constructed in $\mathrm{xerC}^{+}$strains were enriched for suppressing insertions, the majority of insertions $(\sim 85 \%)$ were not suppressing. Five insertions of this type have been mapped (Fig. 1). Southern hybridization was once again used to determine the sizes of the chromosomal restriction fragments in which insertion had occurred, and the possible regions of insertion were then identified on the physical map (K. Rudd, pers. comm.). These sites were subsequently confirmed by cotransduction with closely linked Tn10 insertions (Singer et al. 1989).

We have also used the dif transposon to isolate additional insertions that were in the terminus region but that were not at the dif site and were nonsuppressing. These libraries were made in a xerC mutant, to avoid enrichment of suppressing insertions, and transductions was made to strains that contained various Tn10 insertions to simplify identification of insertions in particular regions. The insertions at $1487,1491,1625$, and $1632 \mathrm{~kb}$ were obtained in this fashion (Fig. 4B).

An insertion at $1539 \mathrm{~kb}$ was isolated by a somewhat different procedure. The library was constructed in a strain that contained a $155-\mathrm{kb}$ dif deletion, $\Delta 2035$ (Fig. 4A). This deletion was formed by homologous recombination between Tn 10 insertions at 1543 and $1698 \mathrm{~kb}$; a Tn10 consequently remained at the site of the deletion. Cotransduction of $\mathrm{Tc}^{\mathrm{r}}$ and $\mathrm{Km}^{\mathrm{r}}$ was then used to identify insertions that were near the ends of the deletion. The original isolate of the insertion at $1539 \mathrm{~kb}$ also contained $\Delta 2035$, and this strain was suppressed for the Dif phenotype. Transduction was then used to separate the insertion from the deletion. The insertion at $1539 \mathrm{~kb}$ was not suppressing when combined with smaller dif deletions, such as $\Delta$ dif::cam 2771 or $\Delta$ dif::spc3269 (Fig. 4A).

The insertions described in the preceding sections were all isolated in derivatives of W3110, which contains 

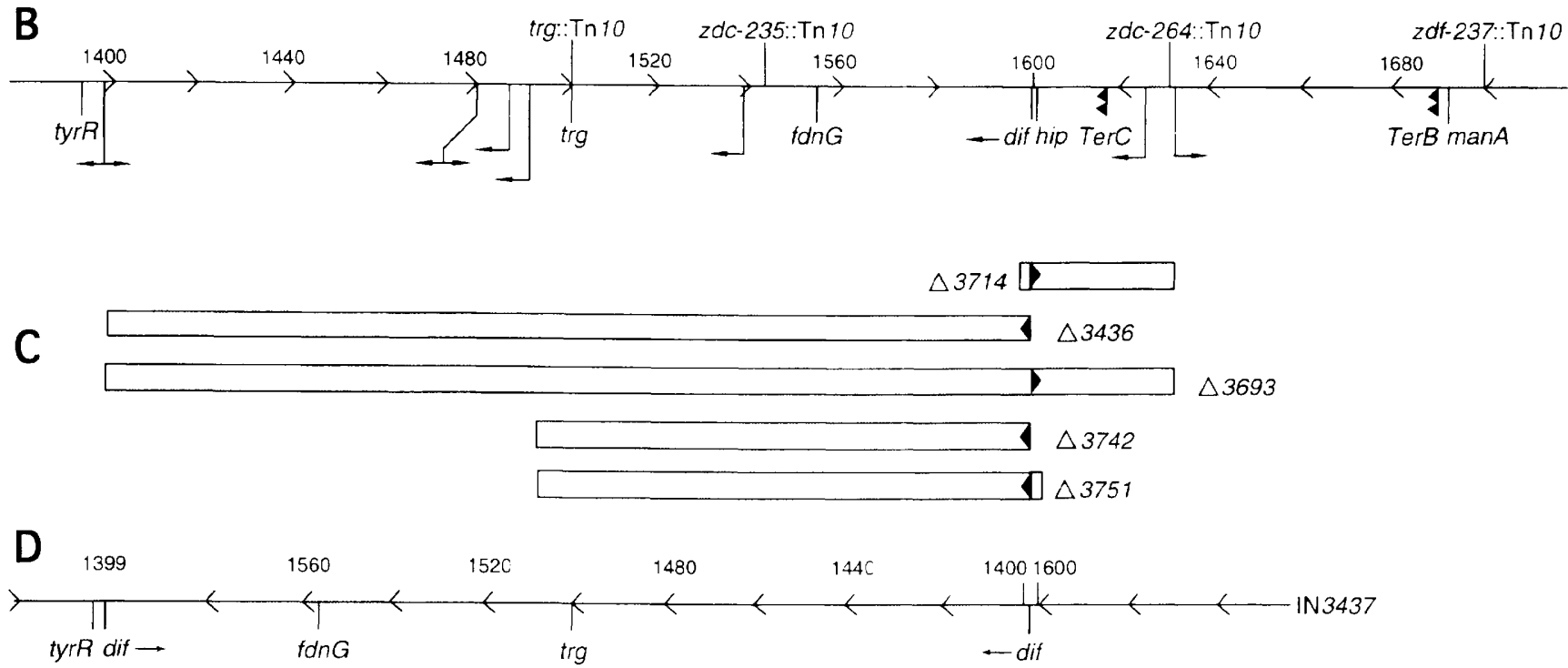

Figure 4. Map of the terminus region. $(A \mid$ dif deletions used in these studies. $|B|$ Physical coordinates in the terminus region, showing relevant Tn10 insertions and loci. The location and orientation of nine Tndif insertions, none of which suppress the Dif phenotype, are indicated by arrows. The physical coordinates are indicated by chevrons every $20 \mathrm{~kb}$, which also indicate the proposed polarity of the terminus region. (C) Deletions formed by recombination between dif sites. All of these suppress the Dif phenotype. (D) Diagram indicating how the inversion of the terminus region in PK3437 shifts the center of this polarity to $1399 \mathrm{~kb}$. The complete designations for the dif insertions, from left to right, are as follows: $\Omega(1399 \mathrm{~kb}:: \leftarrow$ dif-kan)3321, $\Omega(1399 \mathrm{~kb}::$ dif $\rightarrow$ kan $) 3381$,

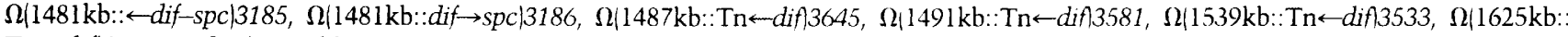
Tn $\leftarrow$ dif $) 3642$, and $\Omega(1632 \mathrm{~kb}::$ Tndif $\rightarrow \mid 3646$.

IN $(r r n D-r r n E) 1$. Comparable results with respect to suppression or nonsuppression have been obtained when these insertions were tested in strains with a wild-type choromsome structure.

\section{Targeted insertions at $1399 \mathrm{~kb}$}

All of the suppressing insertions obtained in strains related to W3110 mapped near the normal dif site at 1599 $\mathrm{kb}$, and none were opposite oriC, near $1400 \mathrm{~kb}$. It seemed likely that insertions in this region did not suppress, and consequently they were not present among the suppressed clones picked for further characterization. It was also possible, however, that the transposon did not insert into this region or that these suppressing insertions were not apparent on the MacConkey-lactose plates. As an additional test of that location, we have constructed insertions of the 231-bp sequence in plasmids and used linear transformation to insert the dif sequence at 1399 $\mathrm{kb}$. Both orientations of dif have been isolated, and for the insertions shown in Figure 4B, kan and dif are oriented with respect to each other as shown in Figure 2. An additional set of insertions was obtained at $1399 \mathrm{~kb}$, in which dif and kan were oriented toward each other $(\mathrm{P}$.
Kuempel, unpubl.). None of these insertions suppressed the Dif phenotype.

\section{Recombination at dif sites}

We reported previously that dif sites in the terminus region, whether suppressing or nonsuppressing, recombined in a RecA-independent fashion with a dif-containing plasmid (pPK 706) at a frequency of $\sim 2 \times 10^{-2}$ (Tecklenburg et al. 1995). A similar frequency was observed for the two insertions at $1399 \mathrm{~kb}$ (Fig. 4B), which are the most distant, nonsuppressing sites that we have isolated in the terminus region. Sites located outside the terminus region, at 3321,3856 , and $4519 \mathrm{~kb}$ (Fig. 1) also recombined with pPK706, although the frequency was $\sim 10^{-3}$ (P. Kuempel, unpubl.).

The nonsuppressing sites in the terminus region could also recombine with other dif sites, both suppressing and nonsuppressing. As we have reported previously, this recombination forms deletions or inversions, it is XerCdependent, and it can generate hybrid, functional sites (Tecklenburg et al. 1995). With the more extensive set of ectopic sites reported here, it has been possible to determine what is required for a fully functional site. 
Examples of some of these rearrangements are provided by studies done with the insertions at $1399 \mathrm{~kb}$ and dif $^{+}$. A "double-dif" strain, containing dif ${ }^{+}(1599 \mathrm{~kb})$ and a dif insertion at $1399 \mathrm{~kb}$ as direct repeats, was stable as a $x e r C$ mutant. Once transduced to $x e r C^{+}$, however, the intervening $200 \mathrm{~kb}$ of DNA was rapidly lost through recombination between the dif sequences, and strains with the resulting deletion $(\Delta 3436$; Fig. $4 \mathrm{C})$ were suppressed. Southern hybridizations demonstrated that strains with this deletion contained the expected fusion fragment (Fig. 5). A variation of this double-dif strain was constructed that contained $\mathrm{dif}^{+}$and an insertion at 1399 $\mathrm{kb}$ as inverted repeats. Both orientations of the intervening DNA were present in $\mathrm{xerC}^{+}$cells grown for 50 gen-

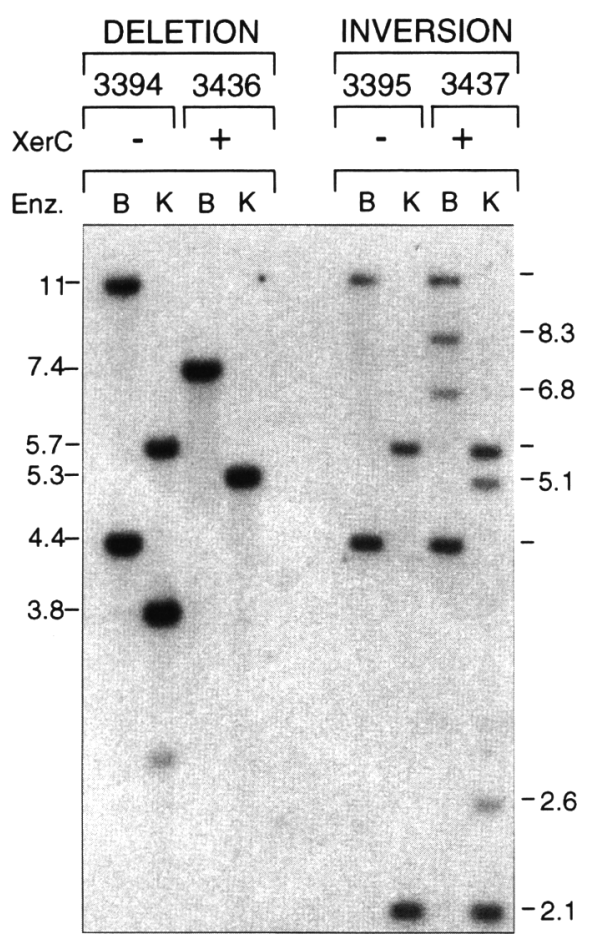

Figure 5. Southern hybridization of double dif strains and their progeny. DNA from xerC $(-)$ and $\operatorname{xerC}^{+}|+|$strains was digested with $B g I I(B)$ and $K p n I(K)$ and probed with the 33-bp dif sequence. Deletion: PK3394 is $\mathrm{xerC} \mathrm{dif}{ }^{+}$and contains an insertion at $1399 \mathrm{~kb}[\Omega(1399 \mathrm{~kb}:: \leftarrow$ dif-kan $) 3321]$. The insertion at $1399 \mathrm{~kb}$ is in a $4.4-\mathrm{kb} B g I \mathrm{I}$ and a $3.8-\mathrm{kb} K p n I$ fragment, and dif ${ }^{*}$ is in an $11-\mathrm{kb} B l g I$ and a $5.7-\mathrm{kb}$ KpnI fragment. In PK3436, the $\mathrm{xerC}^{+}$derivative of PK3394, recombination between the dif sequences produced a $200-\mathrm{kb}$ deletion. The dif fusion fragment is in a 7.4-kb BglI and a 5.3-kb KpnI fragment. Inversion: PK3395 is $\mathrm{xerC} \mathrm{dif}{ }^{+}$and contains an insertion at $1399 \mathrm{~kb}$. $[\Omega(1399$ $\mathrm{kb}::$ dif $\rightarrow$ kan|3381]. The insertion at $1399 \mathrm{~kb}$ is in a $4.4-\mathrm{kb} B g / \mathrm{l}$ and a $2.1-\mathrm{kb} \mathrm{KpnI}$ fragment, and $d \mathrm{if}^{+}$is in an $11 \mathrm{-kb} \mathrm{Bgll}$ and a 5.7-kb KpnI fragment. In $\mathrm{PK} 3437$, the $\mathrm{xerC}^{+}$derivative of PK3395, inversions are apparent in cells grown for 50 generations. The original dif sites were present, as well as two new dif-containing fragments resulting from the inversion. The new dif site at $1399 \mathrm{~kb}$ is in a 6.8-kb BglI and a 2.6-kb KpnI fragment. The new dif site at $1599 \mathrm{~kb}$ is in an 8.3-kb BglI and a $5.1-\mathrm{kb} K p n \mathrm{I}$ fragment. erations (Fig. 5). Because the intensities of the new difcontaining fragments due to the inversion were less than those of the original fragments, this indicates that the inverted orientation of this $200-\mathrm{kb}$ region was present at a lower frequency than the normal orientation. Previously, we described another strain, that inverted the 118 $\mathrm{kb}$ region between dif $^{+}$and an insertion at $1481 \mathrm{~kb}$ (Tecklenburg et al. 1995). In that strain, both orientations were present at equal frequency.

The insertions have been used to construct a number of other deletions (Fig. 4C). $\$ 3693$ was formed by XerCdependent recombination between dif insertions at 1399 and $1632 \mathrm{~kb}$. Neither of the these insertions was suppressing by itself, but strains containing this $233-\mathrm{kb}$ deletion were suppressed. Several other deletions, which also suppressed the Dif phenotype, have been formed by this procedure. $\$ 3742$ was formed by recombination between $\mathrm{dif}^{+}$and an insetion at $1487 \mathrm{~kb}$, and $\Delta 3751$ was formed by recombination between insertions at 1487 and $1600 \mathrm{~kb}$. Finally, $\Delta 3714$ was formed by recombination between insertions at 1596 and $1632 \mathrm{~kb}$.

\section{Discussion}

The experiments reported here were conducted to obtain further information about the requirements of a functional dif site. Most of these insertions were obtained by means of a dif transposon (Tndif), although some were obtained by linear transformation at a specific site. Based on the properties of these insertions, as well as results reported previously by us and others, the requirements for a suppressing site are as follows.

1. Insertion of a 33-bp sequence is sufficient to suppress the Dif phenotype (Leslie and Sherratt 1995; Techlenburg et al. 1995), although the 231-bp site described here gives higher frequencies of recombination between chromosomal sites.

2. Both orientations of the dif sequence are functional for suppressing the Dif phenotype (Fig. 3; Kuempel et al. 1991; Leslie and Sherratt 19951.

3. Other resolvase sites can suppress the Dif phenotype, when placed at the site of a dif deletion. These include the loxP site of P1 (Leslie and Sherratt 1995) and the psi site of pSC101 (Cornet et al. 1994). The cer site of pColEl did not suppress the phenotype (Leslie and Sherratt 1995).

4. Specific flanking sequences are not required for activity; we have observed suppression through insertion of the 33-bp dif sequence at the site of a deletion that removed $173 \mathrm{~kb}$ from the terminus region (Tecklenburg et al. 1995).

5. Suppressing sites are confined to a small region that surrounds the normal dif site (Fig. 3). Using a procedure that enriched for suppressing insertions of Tndif, the most distant insertion that we isolated was $10 \mathrm{~kb}$ on the counterclockwise side of the normal site. The closest nonsuppressing insertions were located $60 \mathrm{~kb}$ to the counterclockwise side and $26 \mathrm{~kb}$ to the clockwise side (Fig. 4B). 
6. Nonsuppressing insertions, both in the terminus region as well as in the rest of the chromosome, are still functional for certain types of recombination. For example, they readily recombined with a dif site on a plasmid (Leslie and Sherratt 1995; Tecklenburg et al. 1995).

7. It is not necessary that suppressing sites be located opposite the origin of replication. In strains with IN(rrnD-rrnE)1, which shifts the region opposite oriC by $200-1400 \mathrm{~kb}$ on the physical map (Fig. 1), all suppressing insertions were still located near the normal location of dif at $1599 \mathrm{~kb}$ (Fig. 3). It should also be noted that the region at $1400 \mathrm{~kb}$ was also near a terminator site (TerA, $1350 \mathrm{~kb}$ ), which is comparable with dif's normal location with respect to $\operatorname{Ter} C$ (1617 $\mathrm{kb}$. Insertions directed to a site at $1399 \mathrm{~kb}$ did not suppress the Dif phenotype (Fig. 4B).

In spite of these specific requirements for sites that can suppress the Dif phenotype, deletions can apparently convert any terminus region-a nonsuppressing site into suppressing site. In general, removal of DNA between a nonfunctional site and the normal position of dif restored full function to the ectopic site. We have observed the following patterns.

1. One set of deletions removed DNA between dif ${ }^{+}$ $(1599 \mathrm{~kb})$ and nonsuppressing sites located to the left of dif. Examples shown in Figure 4C are $\mathbf{} \mathbf{3 4 3 6}$ formed by recombination between $\mathrm{dif}^{+}$and an insertion at $1399 \mathrm{~kb}$, and $\Delta 3742$, formed by recombination between $\mathrm{dif}^{+}$and an insertion at $1487 \mathrm{~kb}$. A simlar suppressing deletion was formed between iff $^{+}$and an insertion at $1491 \mathrm{~kb}$ (data not shown). All of these deletions can be considered to start at the right-hand side of the ectopic site and extend past dif ${ }^{+}$to the adjacent hip gene at $1599 \mathrm{~kb}$.

2. In another set of deletions that generated suppressing sites, the deletions extend several kilobases past dif $^{+}$. An example shown in Figure $4 \mathrm{C}$ is $\Delta 3751$, formed between insertions at 1487 and $1600 \mathrm{~kb}$. A comparable deletion was formed between insertions at 1491 and $1600 \mathrm{~kb}$ (data not shown). Also, we previously described $\Delta 3546$, formed between insertions at 1481 and $1602 \mathrm{~kb}$ (Tecklenburg et al. 1995). Although all of these deletions suppressed the Dif phenotype, strains harboring these deletions sometimes produced a few filamentous cells.

3. A suppressing site can also be generated by a deletion to the right of dif. $\Delta 3714$ was formed by recombination between insertions at 1596 and $1632 \mathrm{~kb}$ (Fig. 4C).

4. $\Delta 3693$ (Fig. $4 \mathrm{C}$ ) removed $233 \mathrm{~kb}$ of DNA between nonsuppressing sites at 1399 and $1632 \mathrm{~kb}$. Neither site, by itself, suppressed the Dif phenotype, presumably because of some property of the region between the ectopic insertion and the normal location of dif. This region was removed by the $233-\mathrm{kb}$ deletion, and the cells appeared completely normal.

5. Perhaps the most informative insertion is that located at $1539 \mathrm{~kb}$ (Fig. 4B). This insertion is nonsuppressing, but it becomes suppressing when combined with
$د 2035$ (Fig. 4A), which extends to within $4 \mathrm{~kb}$ of the insertion. However, the insertion at $1539 \mathrm{~kb}$ is not suppressing when combined with smaller deletions that removed DNA to the left of $d i f^{+}$, such as $\Delta d i f$ :spc3269 (Fig. 4A). Moreover, although $\Delta 2035$ restored suppression to the insertion at $1539 \mathrm{~kb}$, it had no effect on more distant dif insertions at 1481 and $1399 \mathrm{~kb}$. Apparently, a deletion must extend near an ectopic dif site to remove whatever inhibits proper function of the sites. As a test of this, we have used Tn 10 , situated in $\Delta 2035$, to isolate $\operatorname{Tn} 10$-derived deletions (Bochner et al. 1980) in a strain that also contained an insertion at $1481 \mathrm{~kb}$. Approximately $1 \%$ of these derivatives had a suppressed phenotype. These deletions have not been mapped, but it is likely that they extend farther to the left and end near the ectopic dif insertion, removing sites that blocked its full function.

What model can be proposed to explain this pattern of sites and deletions required for proper dif function? We propose that there is a polarity in the terminus region and that two regions of opposite polarity converge at dif. This is illustrated by the arrows indicating the physical coordinates for the map in Figure 4B. This could be a continuation of polarities observed previously in other parts of the chromosome. For example, the majority of Chi sites (Burland et al. 1993) and transcription units (Brewer 1990) are oriented toward the terminus in the halves of the chromosome replicated in clockwise and counterclockwise directions. However, the polarity could also be caused by presently uncharacterized elements, or by sites that are only present in the terminus region. Based on earlier studies on inversions (Rebollo et al. 1988) and more recent studies on hyperrecombination (Louarn et al. 1994), Louarn and co-workers have also proposed that the terminus is flanked by polar regions. It is likely that these different procedures have all identified the same underlying polarity.

One way to visualize the role of polarity is to imagine that it is related to the processing of newly synthesized DNA into nucleoids. Sites that supercoil DNA into separate domains (Løbner-Olesen and Kuempel 1992), for example, might function in a sequential, polar fashion (Fig. 6). Whatever the nature of this polarity, we propose that the signals and processing that occur in clockwise and counterclockwise directions converge at dif but do not cross it. By being where these polarities converge, dif is in a location that facilitates its function as a resolvase site. Location in this region could possibly increase the frequency or the duration of interactions at dif sites in the sister chromosomes. Our present data indicate that this region has a maximum size of $85 \mathrm{~kb}$, but it is probably considerably smaller. If shifted outside this region, an ectopic site would be surrounded by processing sites of the same polarity, which would place it in a region that impeded proper interaction. Deletions that extend from the ectopic dif site up to or past the normal location of dif would place the site once again in the center of the converging polarities (see Fig. 6). A deletion that ren- 


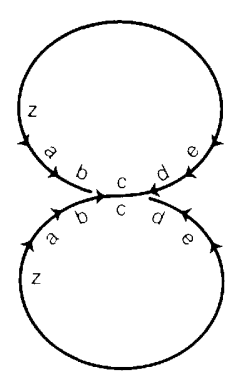

I

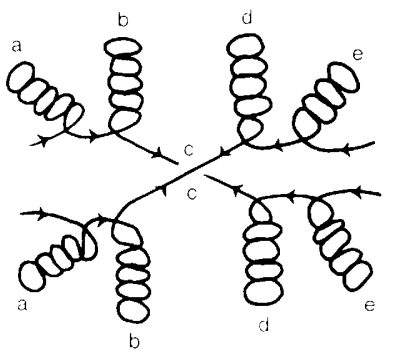

III

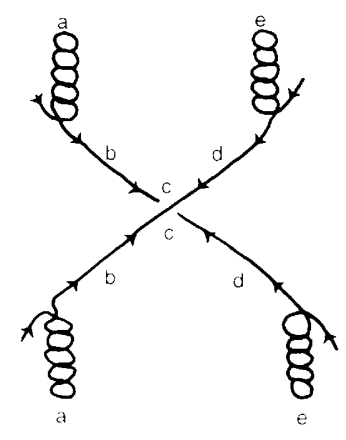

II

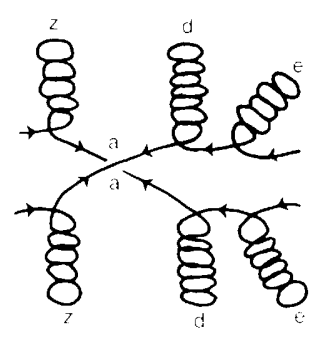

IV
Figure 6. Model for polarity in the terminus region and how this affects dif function. (I) A dimeric chromosome with region c positioned for interaction. The dimer is shown with no nucleoid structure. $(I I)$ The processing of daughter chromosomes into nucleoid domains proceeds in a sequential, polar fashion and is centered at region c. Regions b, c, and d have not yet been processed. (III) Processing from both sides has proceeded up to

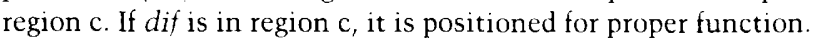
If dif is in another region, such as region a, the sites cannot interact properly. $(I V)$ If a deletion starts near region a and ends at or after c, polarity would now be centered at a, and dif located in this region would be positioned for proper function.

dered one site functional, such as the effect of $\mathbf{2 0 3 5}$ on the insertion at $1539 \mathrm{~kb}$, would not be effective for a more distant site, because that site would still be surrounded by processing sites of the same polarity.

It should be noted that occurrence of functional, ectopic sites indicates that at least with respect to dif function, there is nothing specific about the region at dif. Provided that the new site is at a region were the polarites converge, the ectopic site will be functional. Deletions in the terminus region can be readily tolerated. Insertions and inversions (see below) also would not affect dif function, provided they maintained this polarity around dif.

It is usually assumed that the function of the dif locus is to resolve dimeric chromosomes. It might have other functions, however, such as providing the center about which nucleoid processing occurs, regardless of whether sister chromatid exchange has occurred. A possible model for this has been proposed by Sherratt and co- workers (Sherratt et al. 1995), in which a stable Holliday structure forms at dif shortly after its replication, and resolution does not occur until nucleoid processing is almost complete. Daughter chromosomes joined at dif and polar processing sites flanking that junction could be important for the proper formation of daughter nucleoids.

Although processing into nucleoids must follow replication, the processes do not need to be tightly linked. As proposed by Louarn et al. (1994), the restructuring of newly formed DNA follows its own pattern and occurs even if replication has deviated from the standard pattern. This uncoupling would allow proper processing to occur, even in situations of asymmetric replication in which forks did not meet at dif. This could occur in cells in which the two forks traveled at different rates, or in cells with rearranged chromosomes such as W3110. The Tus/Ter system would ensure that the replication patterns did not become too asymmetric (Hill 1992).

The properties of these polar sites are presently unknown. As described by Cornet et al. (this issue), a site or sites are present in the region near $1617 \mathrm{~kb}$. As we have described above, there are probably many such sites throughout the terminus region.

An apparent contradiction to the above polarity model is strains with inversions of part of the terminus region. Previously, we reported that in cells in which the region between 1481 and $1599 \mathrm{~kb}$ was inverting, there was no preferred orientation (Tecklenburg et al. 1995). Figure 5 shows that cells can also tolerate an inversion of $200 \mathrm{~kb}$ of the terminus region, between 1399 and $1599 \mathrm{~kb}$, although in this case the wild-type orientation was preferred. Figure $4 \mathrm{D}$ shows that, when this region is inverted, the right-hand dif site in PK3437 would be flanked by regions that had the same processing polarity. This seems to violate the polarity model described above. It should be noted, however, that the left-hand dif site at $1399 \mathrm{~kb}$ would now be surrounded by sites of converging polarity, and this could be the functional site when the region is inverted. This raises a number of testable questions concerning recombination at suppressing and nonsuppressing, ectopic dif sites. As one approach for answering some of these questions, we have recently developed a physical assay for monitoring recombination between chromosomal dif sites of these types.

\section{Materials and methods}

\section{Bacterial strains and media}

Bacterial strains are described in Table 1. LB medium (Miller 1992) was used for most liquid cultures, and plates contained LB medium with $1.5 \%$ agar (Difco). MacConkey agar base (Difco) was supplemented with $1.0 \%$ lactose. Phage were grown on LB agar plates in a $2.5-\mathrm{ml}$ top-agar layer of $\mathrm{LB}$ medium with $0.5 \%$ agar. Antibiotic concentrations $(\mu \mathrm{g} / \mathrm{ml})$ were as follows: Kanamycin $(\mathrm{Km}), 100$; tetracycline (Tc), 20; ampicillin (Ap) 100; chloramphenicol $(\mathrm{Cm}) \quad 25$; spectinomycin $\langle\mathrm{Sp}\rangle \quad 100$; trimethoprim (Tp) 10. 


\section{Construction of dif deletions}

The construction of $\Delta$ dif::spc3269 was similar to that of $\Delta$ dif. $\because$ kan2336 (Kuempel et al. 1991), except that a $1.7-\mathrm{kb}$ fragment that contained the spectinomycin resistance gene $|s p c|$ of pHP45- $\Omega$ (Prentki and Krisch 1984) was inserted at the site of the $12-\mathrm{kb}$ deletion in pBS12 to give pOL123. $\Delta$ dif::spc3269 was then crossed into the chromosome by linear transformation to obtain PK3269. Construction of $\Delta$ dif::cam2771 started with pPK999, which is a pUC19 derivative that contains a $7 \cdot \mathrm{kb} P$ P $t \mathrm{I}$ fragment (chromosome coordinates 1594-160l kb). The chloramphenicol resistance gene (cam) was isolated from pACYCl 84 as a 1.3-kb HaeII fragment. The ends were filled in, and the fragment was inserted between the EcoRV and ClaI sites that correspond to chromosome coordinates 1597.7 and $1599 \mathrm{~kb}$ to give pLim 1000. $\Delta$ dif::cam 2771 was then crossed into the chromosome by linear transformation to obtain PK 2771 .

\section{Construction of xerC::tmp}

pMU575, which contains a trimethoprim resistance gene $\mid$ tmp|, was provided by J. Pittard (University of Melbourne, Australial. The tmp gene was removed as a 1.6-kb SphI fragment and cloned into pUC4-Kiss (Pharmacia) that had been digested with SphI. This replaced the kan gene with the tmp gene. tmp was then isolated from this plasmid (pWS1) in a $1.6-\mathrm{kb}$ EcoRI fragment on which the ends had been filled in. pUC18-xerC contained $\mathrm{xerC}$ in an 8.3-kb EcoRI fragment, and after digestion with $B s a \mathrm{BI}, t m p$ was inserted at that site in $x e r C$ to give pWSII. $x e r C:: t m p$ was then inserted into the chromosome by linear transformation to obtain PK3483.

\section{Construction of Tndif}

The 231-bp dif fragment corresponds to positions 1926-2157 kb in the sequence of Black et al. (1991). This fragment, with HindIII and BamHI ends, was isolated from a derivative of pMAK705 (Kuempel et al. 1991) and inserted into pUC18 DNA digested previously with HindIII and BamHI. This construct was then digested with $B a m H I$, and the Tn903 kanamycin resistence gene (kan; Pharmacia) was inserted as a $1.3-\mathrm{kb}$ Bam HI fragment. The dif kan fragment was then isolated from HN5 as a Pvull-EcoRI fragment. After filling in the ends, this was inserted into a $4.8-\mathrm{kb}$ fragment obtained from pNK2859 (Kleckner et al. 1991) by digesting with BamHI and filling in the ends. The product (pHN13) contains the dif kan genes inserted between the 70 -bp inverted repeats. Figure 2 shows the relevant restriction enzyme sites in pHN 13. The polarity arrow for dif indicates the sequence $5^{\prime} \rightarrow 3^{\prime}$ of Black et al. (1991).

\section{Construction of insertions at $1399 \mathrm{~kb}$}

pJPB61 was provided by J.P. Bouche (CNRS, Toulouse, France), and it contained a $4.5 \cdot \mathrm{kb}$ HindIII-BamHI fragment (chromosome coordinates $1396-1401 \mathrm{~kb}$ ) inserted in pBR325. The dif kan fragment was isolated as a PvuII-EcoRI fragment from pHN5. The ends were filled in and the fragment was inserted into the EcoRV site of pJPB61 in both orientations to give pHN7 and pHN8. In the chromosome, this EcoRV site is located at $1399 \mathrm{~kb}$. pHN7 and pHN8 were linearized by digestion with EcoRI and transformed into PK3270. $\Omega(1399 \mathrm{~kb}:: \leftarrow$ difkan) 3321 was derived from pHN7 and $\Omega(1399 \mathrm{~kb}::$ dif $\rightarrow$ kan $\mid 3381$ was derived from $\mathrm{pHN} 8$.

\section{Transposon libraries}

Single colonies of strains containing pHN13 (PK347) or PK3512) were inoculated into LB medium containing $10^{-3} \mathrm{M}$ isopropyl $\beta$-D-thiogalactoside (IPTG) and $100 \mu \mathrm{g} / \mathrm{ml}$ of kanamycin and grown to stationary phase. Bacteriophage Pl were then grown on these bacteria in a LB top-agar layer (Miller 1992). The bacteriophage were harvested after $16 \mathrm{hr}$ of growth, and a number of separate libraries were constructed to avoid sibs. The Tndif insertions were then transduced into PK3032 or derivatives described in Results. To test for suppression, we first selected $\mathrm{Km}^{\mathrm{r}}$ transductants on LB agar plates containing $100 \mu \mathrm{m} /$ $\mathrm{ml}$ of kanamycin, restreaked once, and then streaked the cells on MacConkey-lactose plates. In lac mutants containing the sfiA: lac $Z$ reporter gene, colonies expressing the Dif phenotype were red, because of expression of the sfiA gene when SOS was induced (Kuempel et al. 1991). The cells in these colonies were filamentous. Suppressed colonies were white and contained normal-length cells.

\section{Southern hybridizations}

Total genomic DNA for hybridization was isolated by an SDS lysozyme method (Ausubel et al. 1987). DNA was digested with the appropriate restriction enzymes, separated by electrophoresis in $0.7 \%$ agarose gels, and transferred to Zeta-Probe membranes $\langle$ Bio-Radl, using standard procedures. After transfer, the membranes were baked for $1 \mathrm{hr}$ at $80^{\circ} \mathrm{C}$. Hybridization was conducted for $16 \mathrm{hr}$ at $55^{\circ} \mathrm{C}$. The sequence of the 33 -bp dif oligonucleotide, which was labeled with $\mid \gamma^{-32}$ P|ATP by phosphorylation with bacteriophage $\mathrm{T} 4$ polynucleotide kinase, has been described /Kuempel et al. 19911. After autoradiography, the fragment sizes were compared with the physical map of the relevant region (Rudd 1992, and pers. comm.) to determine the insertion sites. Digestions with EcoRI, EcoRV, BglI, and Pvull were used to identify fragments in which an insertion had occurred. Digestions with KpnI and PstI, which cut in the Tndif transposon (Fig. 2), were used to determine the orientation of the insertions.

\section{Recombination between dif sites}

A number of deletions were formed by XerC-dependent recombination between two dif sites in the same orientation. These double dif strains were constructed in an ilv xerC::cam or ilv xerC::tmp background. If one of the dif sites was dif ${ }^{\circ}$, incorporation of the second site could be obtained by selecting for $\mathrm{Km}^{r}$ |for dif kan insertionsl or $\mathrm{Sp}^{\mathrm{r}}$ (for dif $\mathrm{spc}$ insertions) transductants. If dif kan was to be present at both sites, incorporation of the second site was obtained by selecting for a closely linked marker conferring a different antibiotic resistance, such as a Tn10 insertion. Once both dif sites were present, the strain was transduced to ilv ${ }^{\circ}$, and $x e r C^{\circ}$ cotransductants were identified by loss of $\mathrm{Cm}^{\mathrm{r}}$ or $\mathrm{Tp}^{\mathrm{r}}$. Deletions were initially identified by loss of markers between the two dif sites, and they were subsequently confirmed by Southern hybridizations. Inversions were formed by the same procedure, except dif sites in inverted orientation were used.

\section{Acknowledgments}

We thank John Aron and Heather Szerlong for their capable assistance, and Jean-Michel Louarn's group in Toulouse for frequent exchanges of data and discussions. This research was supported by National Institutes of Health grant GM32968.

The publication costs of this article were defrayed in part by payment of page charges. This article must therefore be hereby marked "advertisement" in accordance with 18 USC section 1734 solely to indicate this fact. 


\section{References}

Ausubel, F., R. Brent, R.E. Kingston, D.D. Moore, I.G. Seidman, J.A. Smith, and K. Struhl, eds. 1987. Current protocols in molecular biology. Wiley Interscience, New York, NY.

Black, D.S., A.J. Kelly, M.J. Mardis, and H.S. Moyed. 1991. Structure and organization of hip, an operon that affects lethality due to inhibition of peptidoglycan or DNA synthesis. I. Bacteriol. 173: 5732-5739.

Blakely, G., S. Colloms, G. May, M. Burke, and D. Sherratt. 1991. Escherichia coli XerC recombinase is required for chromosomal segregation at cell division. New Biol. 3: 789798.

Blakely, G., G. May, R. MuCulloch, L.K. Arciszewska, M. Burke, S.T. Lovett, and D.J. Sherratt. 1993. Two related recombinases are required for site-specific recombination at dif and cer in E. coli K12. Cell 75: 351-361.

Bochner, B.R., H.C. Huang, G.L. Schieven, and B.N. Ames. 1980. Positive selection for loss of tetracycline resistance. I. Bacteriol. 143: 926-933.

Brewer, B.J. 1990. Replication and the transcriptional organization of the Escherichia coli chromosome. In The bacterial chromosome (ed. K. Drlica and M. Riley), pp. 61-83. American Society for Microbiology, Washington, D.C.

Burland, V., G. Plunkett III, D.L. Daniels, and F.R. Blattner. 1993. DNA sequence and analysis of 136 kilobases of the Escherichia coli genome: Organizational symmetry around the origin of replication. Genomics 16: 551-561.

Clerget, M. 1991. Site-specific recombination promoted by a short DNA segment of plasmid Rl and by a homologous segment in the terminus region of the Escherichia coli chromosome. New Biol. 3: 780-788.

Cornet, F.C., I. Mortier, J. Patte, and J.M. Louarn. 1994. Plasmid pSC101 harbors a recombination site, psi, which is able to resolve plasmid multimers and to substitute for the analogous chromosomal Escherichia coli site dif. I. Bacteriol. 176: 3188-3195.

Cornet, F., J. Louarn, J. Patte, and J.M. Louarn. 1996. Restriction of the activity of the recombination site dif to a small zone of the Escherichia coli chromosome. Genes \& Dev. (this issue)

Forro Jr., F. and S.A. Wertheimer. 1960. The organization and replication of deoxyribonucleic acid in thymine-deficient strains of Escherichia coli. Biochim. Biophys. Acta 40: 9-21.

Hill, T.M. 1992. Arrest of bacterial DNA replicaiton. Annu. Rev. Microbiol. 46: 603-633.

Hill, C.W. and B.W. Harnish. 1981. Inversions between ribosomal RNA genes of Escherichia coli. Proc. Natl. Acad. Sci. 78: 7069-7072.

Kleckner, N., J. Bender, and S. Gottesman. 1991. Uses of transposons with emphasis on Tn10. Methods Enzymol. 204: 139-180.

Kohara, Y., K. Akiyama, and K. Isono. 1987. The physical map of the whole Escherichia coli chromosome: Application of a new strategy for rapid analysis and sorting of a large genomic library. Cell 50: 495-508.

Kuempel, P.L., J.M. Henson, L. Dircks, M. Tecklenburg, and D.F. Lim. 1991. Dif, a RecA-independent recombination site in the terminus region of the chromosome of Escherichia coli. New Biol. 3: 799-811.

Leslie, N.R. and D.J. Sherratt. 1995. Site-specific recombination in the replication terminus region of Escherichia coli: Functional replacement of dif. EMBO /. 14: 1561-1570.

Lin, L.L. and J.W. Little. 1988. Isolation and characterization of noncleavable (Ind ${ }^{-}$) mutants of the LexA repressor of Escherichia coli K-12. J. Bacteriol. 170: 2163-2173.
Løbner-Olesen, A. and P.L. Kuempel. 1992. Chromosome partitioning in Escherichia coli. I. Bacteriol. 174: 7883-7889.

Louarn, J., F. Cornet, V. Francois, J. Patte, and J.M. Louarn. 1994. Hyperrecombination in the terminus region of the Escherichia coli chromosome: Possible relation to nucleoid organization. I. Bacteriol. 176: 7524-7531.

Miller, J.H. 1992. A short course in bacterial genetics: A laboratory manual and handbook for Escherichia coli and related bacteria. Cold Spring Harbor Laboratory Press, Cold Spring Harbor, NY.

Prentki, P. and H.M. Krisch. 1984. In vitro insertional mutagenesis with a selectable DNA fragment. Gene 29: 303-313.

Rebollo, J.E., V. Francois, and J.M. Louarn. 1988. Detection and possible role of two large nondivisible zones on the Escherichia coli chromosome. Proc. Natl. Acad. Sci. 85: 93919395.

Rudd, K.E. 1992. Alignment of E. coli DNA sequences to a revised, integrated genomic restriction map. In $A$ short course in bacterial genetics: A laboratory manual and handbook for Escherichia coli and related bacteria, Section 2.43. Cold Spring Harbor Laboratory Press, Cold Spring Harbor, NY.

Sherratt, D.J., L.K. Arciszewska, G. Blakely, S. Colloms, K. Grant, N. Leslie, and R. McCulloch. 1995. Site-specific recombination and circular chromosome segregation. Phil. Trans. R. Soc. Lond. B 347: 37-42.

Singer, M., T.A. Baker, G. Schnitzler, S.M. Deischel, M. Goel, W. Dove, K.J. Jaacks, A.D. Grossman, J.W. Erickson, and C.A. Gross. 1989. A collection of strains containing genetically linked alternating antibiotic resistance elements for genetic mapping of Escherichia coli. Microbiol. Rev. 53: 124.

Tecklenburg, M., A. Naumer, O. Nagappan, and P. Kuempel. 1995. The dif resolvase locus of the Escherichia coli chromosome can be replaced by a 33-bp sequence, but function depends on location. Proc. Natl. Acad. Sci. 92: 1352-1356.

Van Tubergen, R.P. and R.B. Setlow. 1961. Quantitative radioautographic studies on exponentially growing cultures of Escherichia coli. Biophysics I. 1: 589-625. 


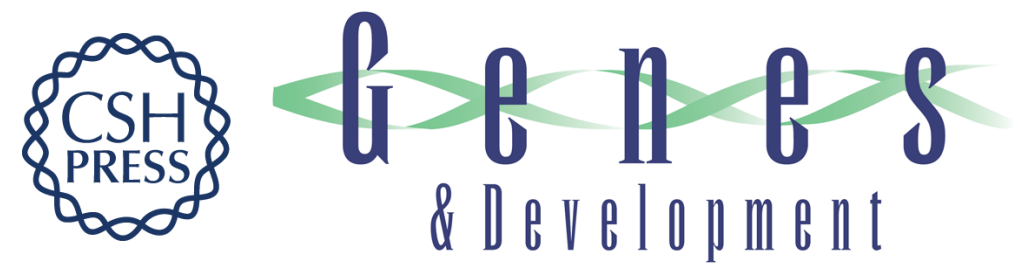

\section{Use of a transposon (Tndif) to obtain suppressing and nonsuppressing insertions of the dif resolvase site of Escherichia coli.}

P Kuempel, A Høgaard, M Nielsen, et al.

Genes Dev. 1996, 10:

Access the most recent version at doi:10.1101/gad.10.9.1162

References This article cites 23 articles, 10 of which can be accessed free at: http://genesdev.cshlp.org/content/10/9/1162.full.html\#ref-list-1

License

Email Alerting Service

Receive free email alerts when new articles cite this article - sign up in the box at the top right corner of the article or click here.

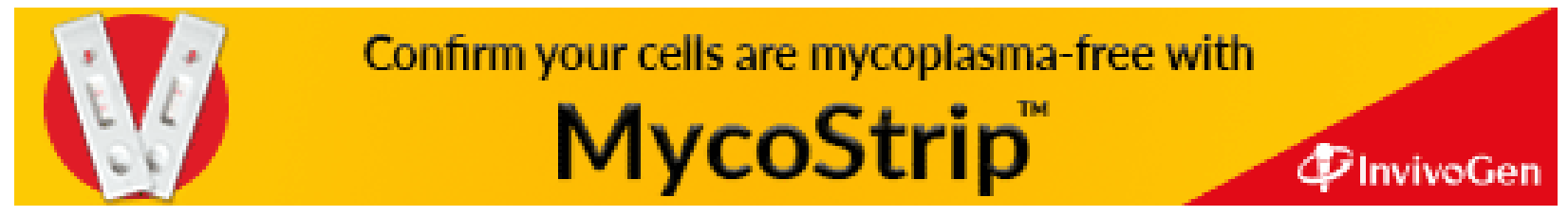

